$16^{\text {th }}$ International Congress of Metrology, 16001 (2013)

DOI: $10.1051 /$ metrology/201316001

(C) Owned by the authors, published by EDP Sciences, 2013

\title{
Feasibility Study for the Development of a Honey-Reference Material
}

\author{
Giovanna Zappa ${ }^{1, \mathrm{a}}$, Laura E. Depero ${ }^{2}$, Laura Borgese ${ }^{2}$, Rosa Giordano and Claudia Zoani ${ }^{1}$ \\ ${ }^{1}$ ENEA - Italian National Agency for New Technologies, Energy and Economic Sustainable Development - Technical \\ Unit "Sustainable Development and Innovation of Agro-industrial System" (UTAGRI) - C.R. Casaccia, Via Anguillarese \\ 301 - 00123 Rome (Italy) \\ ${ }^{2}$ Universisty of Brescia - Dept. of Mechanical and Industrial Engineering - Via Branze 38 - 25123 Brescia (Italy)
}

\begin{abstract}
This paper reports a feasibility study for the development of a Honey-Reference Material (RM). Tests on the possibility to prepare the RM in different physical forms (TQ - liquid, lyophilized powder, pellets of lyophilized honey, encapsulated liquid honey) were carried out on an homogeneous batch of polyfloral honey. The analytical methods to characterize the RM for its elemental profile by TXRF, ICP-AES and ICPMS were developed, employing honey samples of different botanical origin (chestnut, black locust and polyfloral) collected in 20 different Italian Regions. Preliminary results show the feasibility to prepare a Honey-RM to be certified for the elemental profile referred both to nutritional elements and contaminants, as well as to trace elements to be used for the definition of characteristic fingerprints linked to the territorial/botanical product origin. Also the preparation of Honey-RMs in pellet form or encapsulated to be employed as Single Use-RMs turned out to be really feasible.
\end{abstract}

\section{Introduction}

Food traceability and safety represent key factors for ensuring food quality and protecting consumers' interests. Suitable traceability systems are needed to document the history of a product along the entire supply chain (from primary production to the final use), to protect high quality productions from frauds and sophistications and contain health safety risks. To make objective and measurable food quality and traceability, it is necessary to develop analytical methods useful to detect contaminants potentially coming from any phases of the supply chain and define characteristic markers and patterns for the authenticity verification and origin demonstration.

Honey is a very important and healthy food product defined - according to the EU Dir. 2001/110/EC [1] - as "the natural sweet substance produced by Apis mellifera bees from the nectar of plants or from secretions of living parts of plants or excretions of plant-sucking insects on the living parts of plants, which the bees collect, transform by combining with specific substances of their own, deposit, dehydrate, store and leave in honeycombs to ripen and mature". The Directive also define the main honey types and establish the composition criteria (eg. fructose and glucose content, sucrose content, moisture content, electrical conductivity, free acid content) to be meted when honey is placed on the market or is used in any product intended for human consumption.

Generally, honey composition is closely associated with its botanical origin and the geographical area in with originated, because soil and climate characteristics determine melliferous floral. Therefore trace element (including Rare Earth Elements - REE) contents in honey could give an indication on its geographical origin, other than on environmental pollution [2]. In order to perform authenticity and traceability studies on honey botanical and geographical origin, it is possible to apply many analytical techniques that permit to establish its elemental profile, such as: X-Ray Fluorescence (XRF) and Total Reflection X-Ray Fluorescence (TXRF), Inductively Coupled Plasma Atomic Emission Spectroscopy (ICPAES), Inductively Coupled Plasma-Mass Spectroscopy (ICP-MS), and Instrumental Neutron Activation Analysis (INAA). These techniques allow to determine trace elements (including rare earth elements) to be used for the definition of characteristic fingerprints basing on which it is possible to demonstrate the territorial and/or botanical product origin $[3,4]$. Chemical analysis must be then combined with chemometric analysis for basic pattern recognition and honey classification according to the elemental profiles $[5,6]$.

\footnotetext{
a Corresponding author: giovanna.zappa@enea.it
} 
The application of the metrological rules to chemical and biological measurements requires more and more a wide availability of Reference Materials (RMs), which constitute very often the only way for establishing metrological traceability of the measurement results and for quantifying measurement uncertainty. Food sector needs constant update and wide choice of RMs because of the employment of new technologies (i.e. biotechnologies in production, irradiation for preservation, etc.) and growing and diversification of substances to analyse. The Agrofood sector is affected by a lack of RMs, especially RMs to be employed for improving Mediterranean products, assuring origin and traceability of products and raw materials, as well as for carrying out quality and safety controls and for verifying the reliability of chemical and biological measurements. In particular, by examining the current world-wide production of RMs for food analysis, we can highlight that no Honey-RMs are available. Only FAPAS currently distribute a Quality Control Material of honey with an assigned value of chloramphenicol and in recent years a few of feasibility studies for the preparation of honey RMs was conducted $[7,8]$

According to BCR and ISO Guides [9,10], a feasibility study for the preparation of a Honey-RM was conducted. With this purpose, test-batches of honeys similar to those intended to become the Certified Reference Material were prepared both in liquid and in lyophilized form. Usually agrofood RMs are prepared in lyophilized form so to guarantee their stability. Thanks to its high sugar content, honey is very stable also as it is (TQ) in liquid form. However, honey in liquid form can easily undergo segregation phenomena (especially due to crystallization), resulting in a loss of material homogeneity. In addition it is rather difficult to pick up an appropriate test portion and especially to perform precise weighing from liquid honey. Therefore, with the aim to make easier the material handling, as well as to improve the performances of the RM itself, the possibilities to prepare the honey-RM in lyophilized form and as Single Use-RM were evaluated. In particular, the feasibility to prepare the material both in pellet form - as already suggested for other RMs [11,12] - and as capsules of pre-weighed liquid honey was examined. Honey samples were submitted to chemical characterization by applying spectroscopic and mass spectrometry techniques for the definition of the elemental profile. In particular, the possibility to certify the Honey-RM for the elemental composition in terms of macro-, micro- and trace elements were examined by applying the following analytical techniques: TXRF, ICP-AES and ICP-MS. These techniques - other than the determination of elemental fingerprints for origin demonstration - allow the determination (also simultaneously) of both nutritional elements (e.g.: Fe, Zn, $\mathrm{Cu}$ ), and environmental contaminants (e.g.: $\mathrm{Pb}, \mathrm{Cd}, \mathrm{Ni}$, $\mathrm{V})$, as well as contaminants from transformation processes, storage and packaging. Finally the possibility to accompany the RM with information values to be employed in traceability studies (both on botanical and geographic origin) was evaluated.

\section{Materials and Methods}

The feasibility study focused both on tests to develop the $\mathrm{RM}$ in different physical forms, and tests to evaluate the applicability of the different analytical techniques in determining the elemental composition.

The studies about the RM physical form were carried out on an homogeneous batch of polyfloral honey.

As concerns chemical characterization, the tests were conducted on chestnut, black locust (robinia) and polyfloral honeys. 32 honey samples (8 chesnut, 9 robinia, 15 polyfloral) collected in 20 different Italian Regions ( 2 from Friuli Venezia Giulia, 2 from Trentino Alto Adige, 3 from Veneto, 2 from Piedmont, 2 from Lombardy, 2 from Liguria, 2 from Emilia Romagna, 2 from Abruzzo, 1 from Molise, 1 from Tuscany, 2 from Lazio, 1 from Umbria, 2 from Marche, 2 from Campania, 1 from Calabria, 1 from Basilicata, 1 from Puglia, 1 from Sicily, 1 from Sardinia) were employed.

All reagents used were of analytical grade. High-purity water (resistivity $>20 \mathrm{M} \Omega \mathrm{cm}$ ) was used in all dilutions. The elemental standard solutions were prepared by diluting stock solutions (TraceCERT ${ }^{\circledR}$ Fluka Standards) of $1000 \mathrm{mg} / \mathrm{l}$.

\subsection{Preparation of the Test Materials}

Test-batches of RM were prepared in the 4 following physical forms:

a) Liquid form (TQ)

b) Lyophilized powder

c) Lyophilized in pellets

d) Encapsulated liquid honey

Lyophilized powder was obtained by a Virtis Advantage apparatus, in the following operative conditions: Product freeze $\mathrm{T}=-35^{\circ} \mathrm{C}(>24 \mathrm{~h})$; Condenser setpoint $=-45^{\circ} \mathrm{C}$; Drying: $\mathrm{T}$ from $-35^{\circ} \mathrm{C}$ up to $+30{ }^{\circ} \mathrm{C}(100 \mathrm{~h}$; Pmin $=6.5$ $\mathrm{Pa}$; Pmax $=19.7 \mathrm{~Pa})$; Post Heat: $\mathrm{T}=30^{\circ} \mathrm{C}, \mathrm{t}=30 \mathrm{~h}, \mathrm{P}=$ 6.5 Pa. Lyophilization was performed on liquid honey at different dilutions (from 1:2 up to 1:10) using high-purity water.

Honey pellets were prepared starting form lyophilized honey. The pellets (13 mm diameter, $1 \div 5 \mathrm{~mm}$ thickness, $0.2 \div 0.9 \mathrm{~g}$ weight) were obtained by a SPEX-SPECAC press applying a load of $2 \times 10^{4} \mathrm{~kg}$.

Honey capsules were prepared filling cellulose capsules with $0.5 \mathrm{~g}$ of liquid honey.

\subsection{TXRF Analysis}

The elemental analysis by TXRF was carried out on liquefied honey. It was obtained by the following procedure: honey was put in a water bath at $70{ }^{\circ} \mathrm{C}$ for 10 min, than $0.5 \mathrm{~g}$ sample was picked up, diluted with $10 \mathrm{ml}$ high-purity water and thoroughly homogenized. Finally, $0.950 \mathrm{ml}$ of the honey-water solution were collected and spiked with $0.050 \mathrm{ml}$ of Ga standard solution as internal standard. TXRF measurements were performed with a Bruker S2 Picofox equipped with Mo tube, operating at $50 \mathrm{kV}$ and $750 \mu \mathrm{A}$. Samples were measured for $600 \mathrm{~s}$. 


\subsection{ICP-AES and ICP-MS Analyses}

\subsubsection{Dissolution of the Test Material}

Honey dissolution was obtained by acid attack in a Microwave High Pressure Digestion System MILESTONE MLS 1200 MEGA. $0.5 \mathrm{~g}$ of honey were completely dissolved by employing $6 \mathrm{ml}$ concentrated $\mathrm{HNO}_{3} 69.9 \%_{\mathrm{v} / \mathrm{v}}+2 \mathrm{ml}$ concentrated $\mathrm{H}_{2} \mathrm{O}_{2}\left(30 \% \%_{\mathrm{v} / \mathrm{v}}\right)$ and applying the following digestion cycle: $1 \mathrm{~min}$ for $250 \mathrm{~W}$, $1 \mathrm{~min}$ at $0 \mathrm{~W}, 5 \mathrm{~min}$ at $250 \mathrm{~W}, 5 \mathrm{~min}$ at $400 \mathrm{~W}, 5 \mathrm{~min}$ at $600 \mathrm{~W}$ and $10 \mathrm{~min}$ vent (with free pressure rise). The obtained solutions were filled up to the $25 \mathrm{ml}$ final volume with high-purity water in PP flasks and submitted to ICP-AES and ICP-MS analysis. Process blank solutions were prepared and submitted to elemental analysis under identical conditions.

\subsubsection{ICP-AES Analysis}

Solutions obtained by honey dissolution were analyzed using a Varian VISTA MPX (Axial Configuration; simultaneous 1.12 Mpixel CCD detector).

Samples were analyzed at first with a method that allows to perform the study of spectral interferences and the preliminary qualitative analysis (simultaneous detection of the presence of 72 elements). Then quantitative analysis was carried out, under the conditions reported in Table 1.

Table 1. Operating parameters for ICP-AES quantitative analysis

\begin{tabular}{|c|c|c|}
\hline \multicolumn{3}{|c|}{ Instrumental conditions } \\
\hline RF Frequency & \multicolumn{2}{|c|}{$40 \mathrm{MHz}$ (free running, air-cooled) } \\
\hline RF Power & \multicolumn{2}{|l|}{$1.2 \mathrm{~kW}$} \\
\hline $\begin{array}{l}\text { Gas (plasma, } \\
\text { auxiliary, nebulizer) }\end{array}$ & \multicolumn{2}{|l|}{ Argon } \\
\hline Plasma flow & \multicolumn{2}{|l|}{$15.0 \mathrm{l} / \mathrm{min}$} \\
\hline Auxiliary flow & \multicolumn{2}{|l|}{$0.75 \mathrm{l} / \mathrm{min}$} \\
\hline Nebulizer flow & \multicolumn{2}{|l|}{$0.82 \mathrm{l} / \mathrm{min}$} \\
\hline Replicates & \multicolumn{2}{|l|}{5} \\
\hline \multicolumn{3}{|c|}{ Analytical wavelengths (nm) } \\
\hline $\mathrm{Al}-396.152 \mathrm{~nm}$ & $\mathrm{~K}-766.491 \mathrm{~nm}$ & $\mathrm{Rb}-780.026 \mathrm{~nm}$ \\
\hline As $-188.980 \mathrm{~nm}$ & $\mathrm{Li}-670.783 \mathrm{~nm}$ & $\mathrm{~S}-181.972 \mathrm{~nm}$ \\
\hline B - $249.772 \mathrm{~nm}$ & $\mathrm{Mg}-279.553 \mathrm{~nm}$ & $\mathrm{Sb}-206.834 \mathrm{~nm}$ \\
\hline $\mathrm{Ba}-455.403 \mathrm{~nm}$ & $\mathrm{Mn}-257.610 \mathrm{~nm}$ & $\mathrm{Si}-251.611 \mathrm{~nm}$ \\
\hline $\mathrm{Ca}-396.847 \mathrm{~nm}$ & $\mathrm{Na}-589.592 \mathrm{~nm}$ & $\mathrm{Sr}-407.771 \mathrm{~nm}$ \\
\hline $\mathrm{Cr}-267.716 \mathrm{~nm}$ & $\mathrm{Ni}-231.604 \mathrm{~nm}$ & $\mathrm{Ti}-334.941 \mathrm{~nm}$ \\
\hline $\mathrm{Cu}-327.395 \mathrm{~nm}$ & $\mathrm{P}-213.618 \mathrm{~nm}$ & $\mathrm{~V}-311.837 \mathrm{~nm}$ \\
\hline $\mathrm{Fe}-238.204 \mathrm{~nm}$ & $\mathrm{~Pb}-220.353 \mathrm{~nm}$ & $\mathrm{Zn}-206.200 \mathrm{~nm}$ \\
\hline
\end{tabular}

\subsubsection{ICP-MS Analysis}

Solutions obtained by honey dissolution were analyzed using a Bruker Aurora M90 (90 degree ion mirror ion optics; Collision Reaction Interface). The isotopes to be investigated were selected basing on potential interferences and relative abundances. In order to exclude the presence of interferences, for some elements the method was optimized by analyzing more isotopes (eg.: Rb85 and Rb87; Sr88 and Sr87; Nd142, Nd146 and Nd144; Sm147, Sm148 and Sm152; Gd157 and Gd154; Er166 and Er168; Yb170 and Yb172), even applying correction equations with respect to isotopes of other elements potentially interfering. For lead, considering the great variability of the relative abundances of its isotopes in nature, a mathematical combination of the results obtained for the three stable isotopes $\mathrm{Pb} 206, \mathrm{~Pb} 207$ and $\mathrm{Pb} 208$ was employed. The dwell time was optimized for each element in order to obtain the best sensitivity. Table 2 shows the analyzed isotopes and the instrumental conditions for the ICP-MS analysis. For As and Se a specific method was developed to reduce interferences by using the Collision Reaction Interface (Skimmer gas: $\mathrm{H}_{2}$; Skimmer flow $=60 \mathrm{ml} / \mathrm{min}$ ); the potentials of ion optics, Ar flows and dwell time (100 ms) were appropriately optimized. As internal standard, Y89 (except when Y was an analyte) and In115 were employed.

Table 2. Operating parameters for ICP-MS analysis (without CRI)

\begin{tabular}{|c|c|c|c|c|c|}
\hline \multicolumn{6}{|c|}{ Instrumental conditions } \\
\hline \multirow{4}{*}{\multicolumn{2}{|c|}{$\begin{array}{l}\text { Gas flow } \\
\text { parameters } \\
\text { (Argon) }\end{array}$}} & \multicolumn{2}{|c|}{ Plasma flow } & \multicolumn{2}{|c|}{$16.50 \mathrm{l} / \mathrm{min}$} \\
\hline & & \multicolumn{2}{|c|}{ Auxiliary flow } & \multicolumn{2}{|c|}{$2.00 \mathrm{l} / \mathrm{min}$} \\
\hline & & \multicolumn{2}{|c|}{ Nebulizer flow } & \multicolumn{2}{|c|}{$1.00 \mathrm{l} / \mathrm{min}$} \\
\hline & & \multicolumn{2}{|c|}{ Sheat flow } & \multicolumn{2}{|c|}{$0.20 \mathrm{l} / \mathrm{min}$} \\
\hline \multicolumn{2}{|c|}{ Plasma power } & \multicolumn{2}{|c|}{ RF power } & \multicolumn{2}{|c|}{$1.40 \mathrm{~kW}$} \\
\hline \multirow{2}{*}{\multicolumn{2}{|c|}{$\begin{array}{l}\text { Sample } \\
\text { introduction }\end{array}$}} & \multicolumn{2}{|c|}{ Sampling depth } & \multicolumn{2}{|c|}{$6.50 \mathrm{~mm}$} \\
\hline & & \multicolumn{2}{|c|}{ Pump rate } & \multicolumn{2}{|c|}{$4 \mathrm{rpm}$} \\
\hline \multirow{5}{*}{\multicolumn{2}{|c|}{ Ion optics }} & \multicolumn{2}{|c|}{$\begin{array}{l}\text { First extraction lens } \\
\text { Second extraction lens } \\
\text { Third extraction lens } \\
\text { Corner lens }\end{array}$} & \multicolumn{2}{|c|}{$\begin{array}{l}-1.00 \mathrm{~V} \\
-169.00 \mathrm{~V} \\
-202.00 \mathrm{~V} \\
-212.00 \mathrm{~V}\end{array}$} \\
\hline & & \multicolumn{2}{|c|}{ Mirror lens left } & \multicolumn{2}{|c|}{$44.00 \mathrm{~V}$} \\
\hline & & \multicolumn{2}{|c|}{ Mirror lens right } & \multicolumn{2}{|c|}{$22.00 \mathrm{~V}$} \\
\hline & & \multicolumn{2}{|c|}{ Mirror lens bottom } & \multicolumn{2}{|c|}{$37.00 \mathrm{~V}$} \\
\hline & & \multicolumn{2}{|c|}{ Fringe bias } & \multicolumn{2}{|c|}{$-2.50 \mathrm{~V}$} \\
\hline & & \multicolumn{2}{|c|}{ Scan mode } & \multicolumn{2}{|c|}{ Peak hopping } \\
\hline & & \multicolumn{2}{|c|}{ Dwell time } & $10 \div 6$ & $0 \mathrm{~ms}$ \\
\hline Quadru & ipole scan & Points & per peak & 1 & \\
\hline & & Scans/1 & Replicate & 20 & \\
\hline & & Replica & ites/Sample & 5 & \\
\hline Analyz & ed isotope & & & & \\
\hline As75 & $\mathrm{Cu} 65$ & In115 & $\mathrm{Pb}(206,207,208)$ & $\mathrm{Se} 78$ & Th232 \\
\hline Cd11 & Er166 & La139 & Pr141 & Sm147 & Y89 \\
\hline Ce138 & Er168 & Nd142 & Rb85 & Sm148 & Yb170 \\
\hline Ce140 & Eu153 & Nd144 & Rb87 & Sm152 & Yb172 \\
\hline Co59 & Gd154 & Nd146 & Sb12 & Sr87 & V51 \\
\hline $\mathrm{Cr} 53$ & Gd157 & Ni60 & $\mathrm{Sc} 45$ & Sr88 & Zn66 \\
\hline
\end{tabular}

\section{Preliminary results}

Tests carried out showed the feasibility to prepare a Honey-RM in lyophilzed form. The best dilution before lyophilization was 1:10. This preliminary dilution permits both to obtain a more homogeneous material, and to operate under less drastic conditions, by facilitating water sublimation and avoiding difficulties related to the honey tendency to form skin during the drying process. The preparation of the Honey-RM in pellet form turn out to be really feasible. The pellets (Figure 1a) result very compact and hard-wearing, therefore they are proper for the employment as Single Use-RM. The preparation of the encapsulated RM (Figure 1b) to be employed as Single Use-RM (pre-weighed aliquot to be directly inserted into the microwave liner) resulted feasible too. 


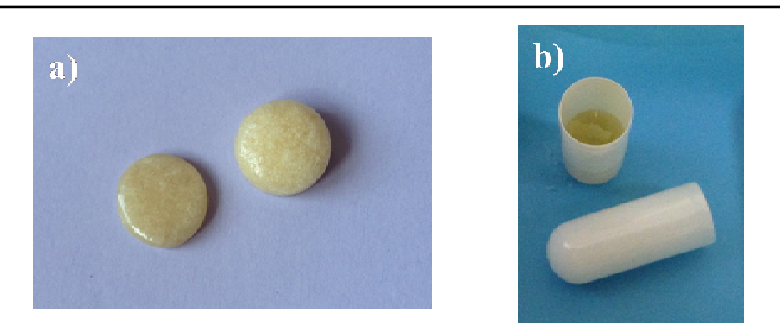

$13 \mathrm{~mm}, \mathrm{~h}=1 \div 5 \mathrm{~mm}, \mathrm{~m}=0.2 \div 0.9 \mathrm{~g}) ;$ b) Encapsulated honey (liquid honey $\mathrm{m}=0.5 \mathrm{~g}$ )

Elemental analysis of the different honey samples by TXRF showed the highest concentrations for $\mathrm{K}, \mathrm{Ca}$ and $\mathrm{Cl}$ (Table 3). In particular, $\mathrm{K}$ is more concentrated in brown honeys (chestnut); $\mathrm{Ca}$ is the second most abundant element, with a higher prevalence for chestnut honeys too; finally, $\mathrm{Cl}$ is the third most abundant element. Also $\mathrm{Fe}$ and $\mathrm{Zn}$ are present in all samples, but in much lower concentrations.

Table 3. Elements in honey by TXRF

\begin{tabular}{|c|c|c|c|c|c|}
\hline & $\begin{array}{c}\mathbf{n}^{\circ} \text { of } \\
\text { analyzed } \\
\text { samples }\end{array}$ & $\begin{array}{c}\mathbf{n}^{\circ} \text { of } \\
\text { samples } \\
\text { < DL }\end{array}$ & $\begin{array}{c}\text { min } \\
\text { quantified } \\
\text { value (mg/kg) }\end{array}$ & $\begin{array}{c}\text { max } \\
\text { value } \\
\text { (mg/kg) }\end{array}$ & $\begin{array}{c}\text { mean } \\
\text { value* } \\
\text { (mg/kg) }\end{array}$ \\
\hline $\mathbf{K}$ & 32 & 0 & 112 & 3740 & 990 \\
\hline $\mathbf{P}$ & 32 & 18 & 3.97 & 14.21 & 7.90 \\
\hline $\mathbf{S}$ & 32 & 18 & 2.48 & 21.05 & 6.53 \\
\hline $\mathbf{V}$ & 32 & 31 & & & 0.60 \\
\hline $\mathbf{B r}$ & 32 & 12 & 0.03 & 1.70 & 0.29 \\
\hline $\mathbf{C a}$ & 32 & 0 & 3.1 & 181 & 51 \\
\hline $\mathbf{C l}$ & 32 & 0 & 12 & 115 & 48 \\
\hline $\mathbf{C r}$ & 32 & 32 & & & \\
\hline $\mathbf{C u}$ & 32 & 13 & 0.04 & 0.61 & 0.20 \\
\hline $\mathbf{F e}$ & 32 & 0 & 0.4 & 3.7 & 1.0 \\
\hline $\mathbf{M n}$ & 32 & 12 & 0.1 & 23 & 4.3 \\
\hline $\mathbf{N i}$ & 32 & 26 & 0.04 & 0.16 & 0.09 \\
\hline $\mathbf{P b}$ & 32 & 10 & 0.02 & 0.25 & 0.11 \\
\hline $\mathbf{R b}$ & 32 & 10 & 0.09 & 193 & 16 \\
\hline $\mathbf{S r}$ & 32 & 29 & 0.07 & 1.7 & 0.72 \\
\hline $\mathbf{Z n}$ & 32 & 1 & 0.1 & 2.6 & 0.6 \\
\hline
\end{tabular}

By ICP-AES and ICP-MS it is possible to determine the content of chemical elements in honey, as well as to define characteristic elemental profiles for honeys of different origin. As an example, concentrations of 25 elements determined by ICP-AES and ICP-MS in robina honey samples are given in Figure 2, while Figure 3 shows the rare earth contents determined by ICP-MS in the same robinia honey samples.

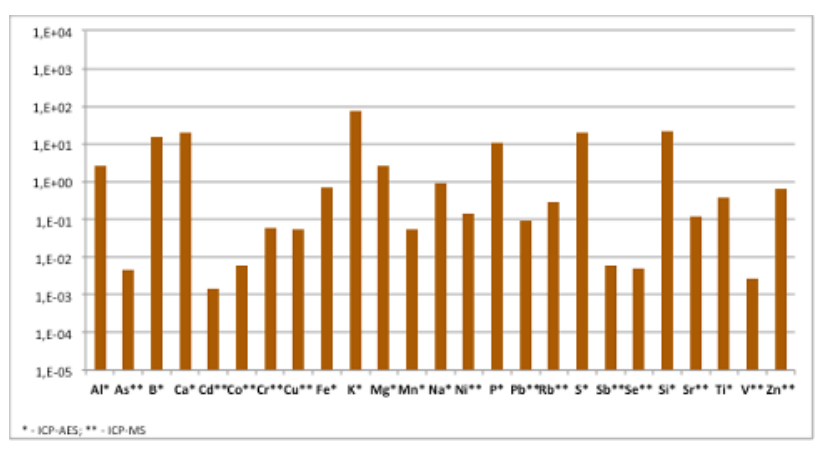

Figure 2. Elemental contents $(\mathrm{mg} / \mathrm{kg})$ in robinia honey by ICPAES and ICP-MS

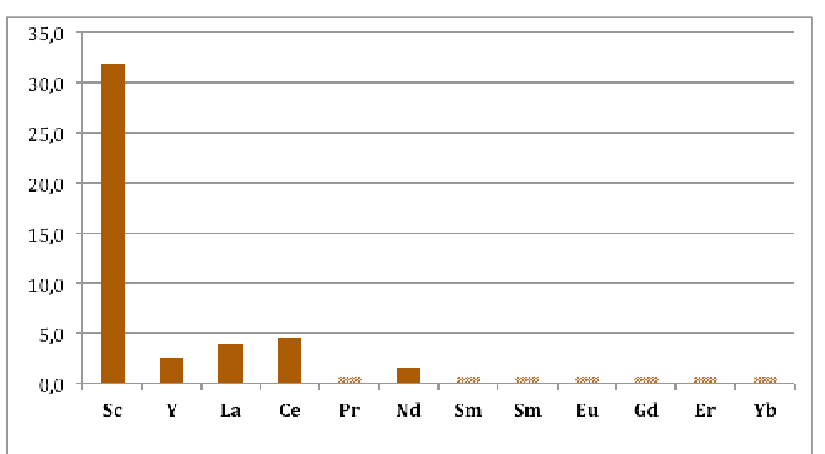

Figure 3. Rare earth contents $(\mu \mathrm{g} / \mathrm{kg})$ in robinia honey by ICPMS

With the aim to evaluate a differentiation of the samples according to their botanical origin and to verify if the floral category corresponded with those indicated by the manufacturer, chemometric analysis of the results obtained by TXRF was performed. The data were submitted to Principal Components Analysis (PCA), which showed 3 clearly distinct groups for chestnut, robinia and polyfloral (Figure 4). For samples at the intersection of the categories chestnut and polyfloral, other available data (eg. sensory analysis and sensorypolarimetry) were evaluated too, showing anomalies from that declared by the manufacturer.

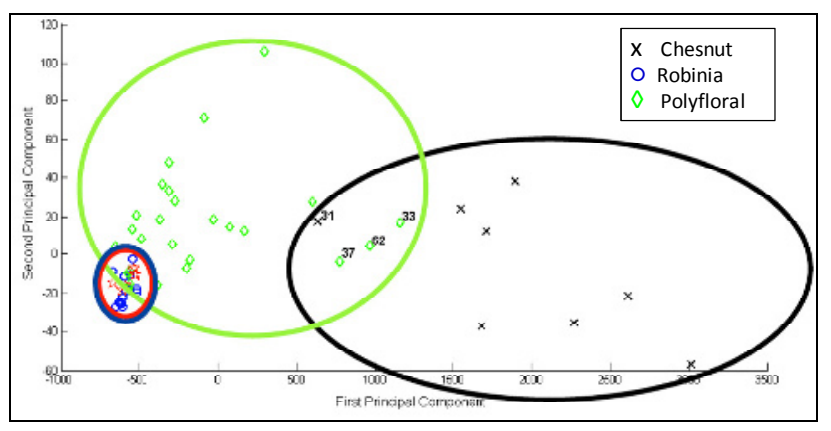

Figure 4. Principal components scatter plot for TXRF analysis

The analyses of the 32 honey samples of different botanical and geographical origin by ICP-AES and ICPMS are currently being completed, but the differences of concentration for some elements among the different samples are already very evident, particularly concerning $\mathrm{Rb}, \mathrm{Sr}$, Th and REE contents.

\section{Conclusions}

The preliminary results show the feasibility of the preparation of a Honey-Reference Material in different physical forms. The preparation of the RM in lyophilized form permits to obtain a more homogeneous materials, thanks to the preliminary dilution with high-purity water. This could be highly advantageous for spiked-RM preparation too. Particularly interesting is the real possibility to prepare Single Use-RMs, both in pellet form and encapsulated. In fact, the use of pellets instead of honey TQ could overtake the difficulties related to the handling and weighing of the material, with great advantages in terms of time and reproducibility. The possibility to prepare encapsulated honey could permit to 
have a Single Use-RM directly from the TQ honey, skipping the lyophilization step, with great advantages in terms of preparation costs and time. It is necessary to consider that lyophilized honey is very highly hygroscopic, therefore it is necessary to operate in humidity-controlled environments (e.g.: rooms with high efficiency dehumidifiers, glove boxes operating in controlled atmosphere) and use appropriate packaging systems. Even for pellets - given the high hygroscopicity - it is necessary to provide for an adequate packaging system to preserve them from humidity.

The procedures for chemical characterization by TXRF, ICP-AES and ICP-MS were developed and established, and the analytical technique most suitable for the determination of each different element was stated (Table 4). TXRF permits to determine halogens, while ICP-AES and ICP-MS allow to determine almost all the elements with a low Detection Limit (DL). On the whole, for almost all the elements almost two different analytical techniques are available, and for some elements ( $\mathrm{Sr}$ and $\mathrm{Zn}$ ) even three techniques.

In RM development, TXRF - not requiring the sample dissolution - is suitable for homogeneity and stability studies, and as a complementary technique for the RM characterization (or certification). ICP-MS permits to determine rare earth elements at very low concentration levels $(\mathrm{DL}=10 \mu \mathrm{g} / \mathrm{l}$ in solution, corresponding to about $0.7 \mu \mathrm{g} / \mathrm{kg}$ in honey), so it can be usefully applied for determining characteristic fingerprints referred to the environment of production or the botanical origin.

The first applications of chemometric analysis to TXRF results allowed to differentiate the honey samples according to their botanical origin, and the applied model permitted to detect anomalies on the honey quality or on manufacturer declarations, only basing on the elemental analysis.

Homogeneity studies and stability studies under luminous and thermal stress are underway. Additional ongoing tests on the definition of elemental fingerprints and further applications of chemometric analysis on the obtained results will allow to demonstrate the real possibility to employ matrix-RMs for traceability studies (both for botanical and geographic origin). The further development of this feasibility study will be to evaluate the possibility to certify the Honey-RM also in terms of environmental organic contaminants and pesticide residues, by applying GC, HPLC and LC-MS techniques. In this way it will be possible to provide a unique RM certified or accompanied by reference or information value on different parameters (nutrients, elemental and organic contaminants, elemental profiles for traceability studies).

\section{References}

1. Council directive 2001/110/EC of 20 December 2001relating to honey (OJ L 10, 12.1.2002, p. 47) + Corrigendum, OJ L 52, 21.2.2007, p. 16 (110/2001)

2. R.G.Pellerano, M.A. Unates, M.A. Cantarelli, J.M. Camina, E.J. Marchevsky. Food Chemistry 134, 578-582 (2012)

3. M. Madejczyk, D. Baralkievicz. Analytica Chimica Acta 617, 11-17 (2008)

4. M. Chudzinska, A. Debska, D. Baralkiewicz. Accred. Qual. Assur., 17: 65-73 (2012)

5. R. Fernandez-Torres, J.L. Pérez-Bernal, M.A. Bello-Lopez, M. Callejon-Mochon, J.C. JimenezSanchez, A. Guiraum-Pérez. Talanta 65, 686-691 (2005)

6. M. Chudzinska, D. baralkievicz. Food and Chemical Toxicology, 48, 284-290 (2010)

7. G. Forte, S. D'Ilio, S. Caroli. Journal of AOAC International, 84, 6 (2001)

8. D.A. Bohm, C.S. Stachel, R. Hackenberg, P. Gowik. Analytica Chimica Acta, 700, 58-62 (2011)

9. Doc. BCR/01/97 - Part A (1997)

10. ISO GUIDE 35 (2006)

11. R. Gatti, P. Sangiorgio, G. Zappa, C. Zoani. RAFA 2009

12. G. Zappa, P. Carconi, R. Gatti, A. D’Alessio, R. Di Bonito, L. Mosiello, C. Zoani. Measurement 42, 14911496 (2009)

Table 4. Most suitable analytical techniques for nutrients and contaminants

\begin{tabular}{|c|c|c|c|c|c|c|c|c|c|c|c|c|c|c|c|c|c|c|c|c|c|c|c|c|c|c|c|c|c|}
\hline & Al & As & B & $\mathbf{B a}$ & $\mathrm{Br}$ & $\mathrm{Ca}$ & Cd & $\mathrm{Cl}$ & Co & $\mathrm{Cr}$ & $\mathbf{C u}$ & $\mathbf{F e}$ & $\mathbf{K}$ & $\mathbf{L i}$ & Mg & Mn & $\mathbf{N a}$ & $\mathbf{N i}$ & $\mathbf{P}$ & $\mathbf{P b}$ & $\mathbf{R b}$ & \begin{tabular}{l|l}
$\mathbf{S}$ & $\mathbf{S}$
\end{tabular} & $\mathbf{S b}$ & $\mathbf{S e}$ & $\mathbf{S i}$ & $\mathbf{S r}$ & $\mathbf{T i}$ & \begin{tabular}{l|l}
$\mathbf{V} 2$ \\
\end{tabular} & $\mathbf{Z n}$ \\
\hline TXRF & & & & & & & & & & & & & & & & & & & & & & & & & & & & & \\
\hline ICP-AES & & & & & & & & & & & & & & & & & & & & & & & & & & & & & \\
\hline ICP-MS & & & & & & & & & & & & & & & & & & & & & & & & & & & & & \\
\hline
\end{tabular}

